\title{
The Moderator Role of Caffeine Intake in Adolescents' Sleep and Health Behaviors
}

\author{
Sílvia Pucci (D) and M. Graça Pereira \\ University of Minho, Braga, Portugal
}

\begin{abstract}
The aim of this study was to examine the relationships among excessive daytime sleepiness, family sleep behaviors, sleep quality, and health behaviors, as well as the role of caffeine intake as a moderator. The study included a sample of 272 adolescents that answered a sociodemographic questionnaire, the Family Sleep Questionnaire, the Pittsburgh Sleep Quality Index, the Modified Excessive Sleepiness Scale, and the Health Behavior Questionnaire. The results revealed that higher caffeine intake was associated with worse sleep quality, worse family sleep behaviors, and fewer healthy behaviors. However, caffeine intake was not associated with excessive daytime sleepiness. In addition, worse sleep quality was associated with worse family sleep behaviors, excessive daytime sleepiness, and fewer healthy behaviors. Worse family sleep behaviors were associated with excessive daytime sleepiness and lower health behaviors. Finally, excessive daytime sleepiness was associated with fewer healthy behaviors. Caffeine intake was a moderator in the relationship between daytime sleepiness and health behaviors. Health professionals (i.e., pediatricians, psychologists, and teachers), when intervening on sleep quality and health behaviors, should assess the adolescent's caffeine intake.
\end{abstract}

\section{ARTICLE HISTORY}

Received 28 March 2017

Accepted 17 December 2018

\section{KEYWORDS}

adolescents; caffeine intake; family sleep behaviors; health behaviors; sleep quality; sleepiness

\section{Introduction}

Adolescence is a developmental period characterized by several changes in biological, social, and psychological life dimensions. During this period, adolescents develop their independence and responsibility for their own health behaviors, and acquire skills to answer psychosocial demands.

Regarding health behaviors, the increasing caffeine consumption by adolescents has often been a reflection of the variety of products containing caffeine far beyond the simple tea and coffee (e.g., soft and energy drinks) with massive advertising to win these audiences (Kristjansson, Sigfusdottir, Allegrante, \& James, 2011). A recent literature review found a high prevalence of caffeine intake among children and adolescents (Temple, 2009), probably due to the fact that caffeine is a legal, easily accessible stimulant. The use of caffeine may be simultaneously associated with activities that compete with sleep, having an impact on daytime sleepiness, in children and adolescents (Calamaro, Mason, \& Ratcliffe, 2009). Poor sleep quality (restricted sleep) has been linked to risk behaviors, as well as mental and psychological illness (Owens \& Adolescent Sleep Working Group, 2014).

The use of technology associated with the consumption of caffeine at night, when the adolescent should be sleeping, often translates into excessive daytime sleepiness. A study found that adolescents use a variety of technological devices such as television, games, computer, text messaging, and mobile, with the simultaneous consumption of caffeine (e.g., energy drinks, coffee, black tea), in order to stay awake longer, in these parallel activities (Calamaro et al., 2009). The medium to high consumption of caffeine doses, in children and adolescents, is around 100 to 400 milligrams, with a negative impact on their health (i.e., poor sleep quality and lower quality of life; Temple, Dewey, \& Briatico, 2010). Also, there are a number of symptoms associated with caffeine withdrawal, particularly decreased alertness/attention, sleepiness, and depressed mood (Juliano \& Griffiths, 2004). Recent literature has found an association between caffeine use and risk behaviors (Temple, 2009; contrary to healthy behaviors), such as nicotine, alcohol, and other drug dependence (Reissig, Strain, \& Griffiths, 2009), sexual risk behaviors, and violence (Von Elm et al., 2007). In a representative self-reported health/well-being assessment of adolescents, in public middle and high school, an association between sleep quality (sleep duration) and risk behaviors (consumption of alcohol and other drugs, violence) was found (Owens, Wang, Lewin, Skora, \& Baylor, 2017).

The way parents and the family, in general, role-model health behaviors for their children (e.g., having breakfast) has also been studied in the literature (Pearson, Biddle, \& Gorely, 2009). In fact, an epidemiological study revealed that the lack of parental flexibility and a rigid family environment were associated with an increased body mass index in adolescents and children (Berge, Wall, Bauer, \& Neumark- 
Sztainer, 2010). Unhealthy behaviors have also been associated with being overweight in adolescents (NeumarkSztainer, Story, Hannan, Perry, \& Irving, 2002). Family environment has been associated not only with health behaviors, but with health behavior outcomes as well. A clinical review found that a disorganized family environment was related to impaired sleep quality (e.g., onset sleep latency, sleep duration) in adolescents and that family sleep behaviors had an impact on adolescents' sleepiness but impaired the adolescents' sleep quality (Bartel, Gradisar, \& Williamson, 2015).

The Stress-Coping Model (Maes, Leventhal, \& de Ridder, 1996) served as the basis for the present study. According to the model, when faced with a stressor (as a chronic disease), individuals assess the situation, taking into consideration disease characteristics, sociodemographic characteristics, and life events that are disease related. This evaluation also takes into account personal requirements and goals that the individual wants to achieve, as well as external (social support, support of health professionals) and internal resources (personality, resilience). To deal with the stress, individuals use certain coping strategies, which in turn will result in social, psychological, and physical consequences. Depending on the nature, etiology, and beliefs about the disease, coping strategies may be used at the same time. In fact, according to the present model, what will determine the evaluation of the situation as stressing and the coping resources used will be the cognitive evaluation process. In a first evaluation, the individual will impute the meaning that the event will have on his/her well-being, performing a stressor analysis, determining how to react. The coping efforts used will depend on what the evaluation has been previously determined as neutral, positive, or negative. In a secondary assessment, what happens is an analysis of the resources that are available to deal with a particular stress situation. Both strategies are related to each other, with the emotional ones being present immediately and later replaced by problem-solving strategies. The success of these strategies depends on how the individual is able to manage the emotional discomfort and the problem causing the discomfort. Thus, according to the model, coping may moderate the relationship between the cognitive/emotional response, the internal resources available and the physical, psychological, social consequences (i.e., quality of sleep and health behaviors). The sociodemographic characteristics, in the present study, included age, gender, and the use of psychoactive substances. Regarding internal resources, the focus was on family sleep behaviors. Caffeine intake, as a coping strategy, was analyzed as a moderator between the sleep disturbance (response)/family sleep behaviors (the internal resource), and the outcome variables: sleep quality and health behaviors.

Contrary to the adult studies with caffeine, the literature regarding caffeine and sleep quality in pediatric populations is scarce (Temple et al., 2010). There is a lack of studies focused on the impact of caffeine intake in adolescents' sleep quality and, therefore, there is a need to understand the effects and impact of caffeine on this population (Bernstein, Carroll, Thuras, Cosgrove, \& Roth, 2002). Therefore, the present study analyzed the relationships among excessive daytime sleepiness, family sleep behaviors, sleep quality, and health behaviors; the moderator role of caffeine intake in the relationship between excessive daytime sleepiness and adolescents' sleep quality/health behaviors; and the moderator role of caffeine intake in the relationship between family sleep behaviors and adolescents' sleep quality/health behaviors. It is expected that caffeine intake will be associated with excessive daytime sleepiness, worse family sleep behaviors, poor sleep quality, and fewer healthy behaviors and that caffeine intake will moderate the relationship between excessive daytime sleepiness and sleep quality/ health behaviors as well as between family sleep behaviors and sleep quality/health behaviors.

\section{Methods}

\section{Description of participants}

This study used a cross-sectional design and a convenience sample of 272 adolescents from two public schools in the North of Portugal in an urban zone. The inclusion criteria were: being an adolescent 12 to 18 years old, having a written parental consent to participate in the study, and being in the class the day of the assessment.

\section{Measures}

Adolescents answered the following instruments adapted to the Portuguese population:

\section{Sociodemographic Questionnaire}

The sociodemographic questionnaire was developed with the purpose to collect demographic and social data. This questionnaire consists of multiple-choice questions addressing adolescents' age, sex, use of medications, consumption of substances with caffeine (soft drinks and energy drinks) and family variables such as education, marital status, alcohol and tobacco consumption, and presence of sleep problems. The consumption of caffeine was assessed through the number of cups of coffee the adolescent consumed per day (1-6 or more).

\section{Howard Family Sleep Questionnaire (HFSQ; Billings, Bangash, \& Berg-Cross 2010)}

This scale assesses the cumulative impact of family dynamics on sleep behavior and includes 52 items that assess physical and cultural aspects of the family environment that influences sleep (e.g., "we respect each other's needs for quiet once one of us goes to bed"; "on weekends, we have no set bedtime"). A higher score indicates worse family sleep behaviors. In this study, Cronbach's alpha for the total scale was .70. 
Pittsburgh Sleep Quality Index (PSQl; Buysse, Reynolds, Monk, Berman, \& Kupfer, 1989)

This instrument assesses sleep quality during the previous month and is composed of 19 items. The PSQI has seven components that evaluate sleep efficiency, daytime dysfunction, sleep disturbances, sleep duration, sleep medication, sleep latency, and sleep quality (e.g., "During the past month, how many times have you had trouble sleeping because (a) could not sleep within 30 minutes; (b) felt cold?"). Higher scores indicate worse sleep quality. In the present study, Cronbach's alpha for the total scale was .60 that is considered acceptable in this context (DeVellis, 1991).

\section{Modified Excessive Sleepiness Scale (MESSA; Billings \& Berg-Cross, 2010)}

This instrument assesses the propensity of adolescents to fall asleep in different situations and is composed of 13 items. (e.g., in the classroom, studying, watching television). A higher score indicates excessive daytime sleepiness. In this study, the Cronbach's alpha for the total scale was .78.

\section{Health Behavior Questionnaire (HBQ; Pereira \& Pedras, 2008)}

This instrument assesses health behaviors in teenagers (e.g., exercising, having a meal with vegetables and fruits, eating regularly, smoking, drinking alcohol). A higher score indicates a higher number of healthy behaviors. In this study, Cronbach's alpha for the total scale was .70.

\section{Procedure}

School principals who agreed to participate offered a class period for students' participation. Data were collected in two urban high schools in the Northern region of Portugal. Participation was voluntary. Prior to the assessment parents received at home information regarding the study. The selfadministered questionnaires were completed during the class period. The study was approved by the Portuguese General Education Committee.

\section{Data analysis}

In order to analyze the relationships between variables, the Pearson coefficient correlation was used. To analyze the moderating role of caffeine intake, the macro PROCESS command in SPSS with bootstrapping version 2.16.1 was used (Hayes, 2013).

The assumptions to perform the moderation analysis were all fulfilled, with the variables being normal and the independent variables (excessive daytime sleepiness, sleep family behaviors, sleep quality) with the dependent variable (health behaviors) being correlated, as well as the moderating variable (caffeine intake) with the independent variables.

Age and sex were controlled as covariates in all the analyses.

\section{Results}

\section{Sample characterization}

The sample included 272 adolescents. Regarding gender, $60 \%$ were girls, $92 \%$ were Caucasian, and $79 \%$ had married parents who lived together. From the total sample, 65\% reported no sleep problems present in the family. Seventyfour percent of fathers and $52 \%$ of the mothers consumed alcohol. The average caffeine intake (measured in number of cups) by adolescents was $4.20(S D=1.31)$. Of the total sample, $38 \%$ of caffeine intake was consumed by adolescents between 12 and 15 years old and $62 \%$ by adolescents between 16 and 18 years old. The sociodemographic characterization of the sample is presented in Table 1 and the descriptive results of the psychological variables are presented in Table 2.

\section{Relationships among excessive daytime sleepiness, Family sleep behaviors, Sleep quality, and health behaviors}

Higher caffeine intake was significantly associated with worse family sleep behaviors and lower sleep quality and fewer healthy behaviors. In addition, lower sleep quality was

Table 1. Descriptive statistics for sociodemographics $(N=272)$.

\begin{tabular}{|c|c|c|c|c|}
\hline Continuous Measure & Min & Max & Mean & $S D$ \\
\hline Age (years) & 12 & 18 & 15.48 & 1.61 \\
\hline Adolescent educational level (number of years) & 0 & 12 & 9.61 & 1.71 \\
\hline Caffeine intake (number of cups) & 0 & 10 & 4.20 & 1.31 \\
\hline Categorical measure & & & & $\%$ \\
\hline \multicolumn{5}{|l|}{ Gender } \\
\hline Boys & & & & 41.5 \\
\hline Girls & & & & 58.5 \\
\hline \multicolumn{5}{|l|}{ Caffeine intake } \\
\hline $12-15$ years old & & & & 38 \\
\hline $16-18$ years old & & & & 62 \\
\hline \multicolumn{5}{|l|}{ Race/ethnicity } \\
\hline Caucasian & & & & 92.1 \\
\hline Others & & & & 7.9 \\
\hline \multicolumn{5}{|l|}{ Parents' marital status } \\
\hline Married & & & & 79.3 \\
\hline Divorced & & & & 14.4 \\
\hline Other & & & & 6.3 \\
\hline \multicolumn{5}{|l|}{ Fathers' educational level } \\
\hline Secondary & & & & 39.5 \\
\hline Elementary & & & & 35.0 \\
\hline Other & & & & 25.5 \\
\hline \multicolumn{5}{|l|}{ Mothers' educational level } \\
\hline Secondary & & & & 32.8 \\
\hline Elementary & & & & 32.5 \\
\hline Others & & & & 34.7 \\
\hline \multicolumn{5}{|l|}{ Family member with sleep problems } \\
\hline No & & & & 64.5 \\
\hline Yes & & & & 35.5 \\
\hline \multicolumn{5}{|l|}{ Fathers' alcohol consumption } \\
\hline No & & & & 26.2 \\
\hline Yes & & & & 73.8 \\
\hline \multicolumn{5}{|l|}{ Mothers' alcohol consumption } \\
\hline No & & & & 48.3 \\
\hline Yes & & & & 51.7 \\
\hline \multicolumn{5}{|l|}{ Fathers' tobacco smoking } \\
\hline No & & & & 69.0 \\
\hline Yes & & & & 31.0 \\
\hline \multicolumn{5}{|l|}{ Mothers' tobacco smoking } \\
\hline No & & & & 84.4 \\
\hline Yes & & & & 15.6 \\
\hline
\end{tabular}


associated with worse family sleep behaviors, excessive daytime sleepiness, and fewer healthy behaviors. Excessive daytime sleepiness and worse family sleep behaviors were associated with fewer health behaviors (Table 3).

\section{Use of caffeine as moderator in the relationship between excessive daytime sleepiness and sleep quality/ Health behaviors}

The use of caffeine intake was not a moderator in the negative relationship between daytime sleepiness and sleep quality $F(3,266)=10.21, p<.001, \beta=-.019,95 \%$ CI $[-.052$, $-.014], t=-.113, p=.256$.

Table 2. Descriptive statistics for the psychological variables $(N=272)$.

\begin{tabular}{lrc}
\hline Variable & Mean & $S D$ \\
\hline Family sleep behaviors & 1.31 & .517 \\
Sleep quality & 4.47 & 2.37 \\
Excessive Daytime Sleepiness & 12.48 & 6.22 \\
Health behaviors & 59.43 & 7.36 \\
\hline
\end{tabular}

Table 3. Results of Pearson's coefficient correlation between psychosocial variables.

\begin{tabular}{lccccr}
\hline Measure & 1 & 2 & 3 & 4 & 5 \\
\hline 1. Caffeine intake & - & & & & \\
2. Sleep quality & $.177^{* *}$ & - & & \\
3. Family sleep behaviors & $.240^{* * *}$ & $.346^{* * *}$ & - & \\
4. Excessive daytime sleepiness & .049 & $.277^{* * *}$ & $.235^{* * *}$ & - \\
5. Health behaviors & $-.225^{* * *}$ & $-.270^{* * *}$ & $-.543^{* * *}$ & $-.170^{* *}-$ \\
\hline$* * p<.01 . * *$ & & & &
\end{tabular}

** $p<.01$. ${ }^{* * *} p<.001$.
The use of caffeine intake was a moderator in the negative relationship between daytime sleepiness and health behaviors regardless of sex and age (both not significant) $F$ $(5,263)=3.22, p<.001, \beta=.181,95 \%$ CI $[.075, .286]$, $t=3.38, p=.0012$, explaining $5.7 \%$ of the variance with an effect size of 0.61 (i.e., there was a negative relationship between daytime sleepiness and health behaviors when caffeine intake was lower), $\beta=-.3109,95 \%$ CI $[-.501$, $-.1204], t=-3.21, p<.001$ (Figure 1).

\section{Use of caffeine as moderator in the relationship between family sleep behaviors sleep quality/and health behaviors}

The use of caffeine intake was not a significant moderator in the negative relationship between family sleep behaviors and sleep quality, $F(3,241)=10.21, p<.001, \beta=-.019$, $95 \%$ CI $[-.052, .014], t=-.1 .13, p=.256$ nor in the negative relationship between family sleep behaviors and health behaviors, $F(3,221)=35.09, p<.001, \beta=.024,95 \% \mathrm{CI}$ $[-.045, .094], t=.687, p=.493$.

\section{Discussion}

Higher caffeine intake was significantly associated with worse family sleep behaviors and lower sleep quality and fewer healthy behaviors. In addition, lower sleep quality was associated with worse family sleep behaviors, excessive daytime sleepiness, and fewer healthy behaviors. Excessive

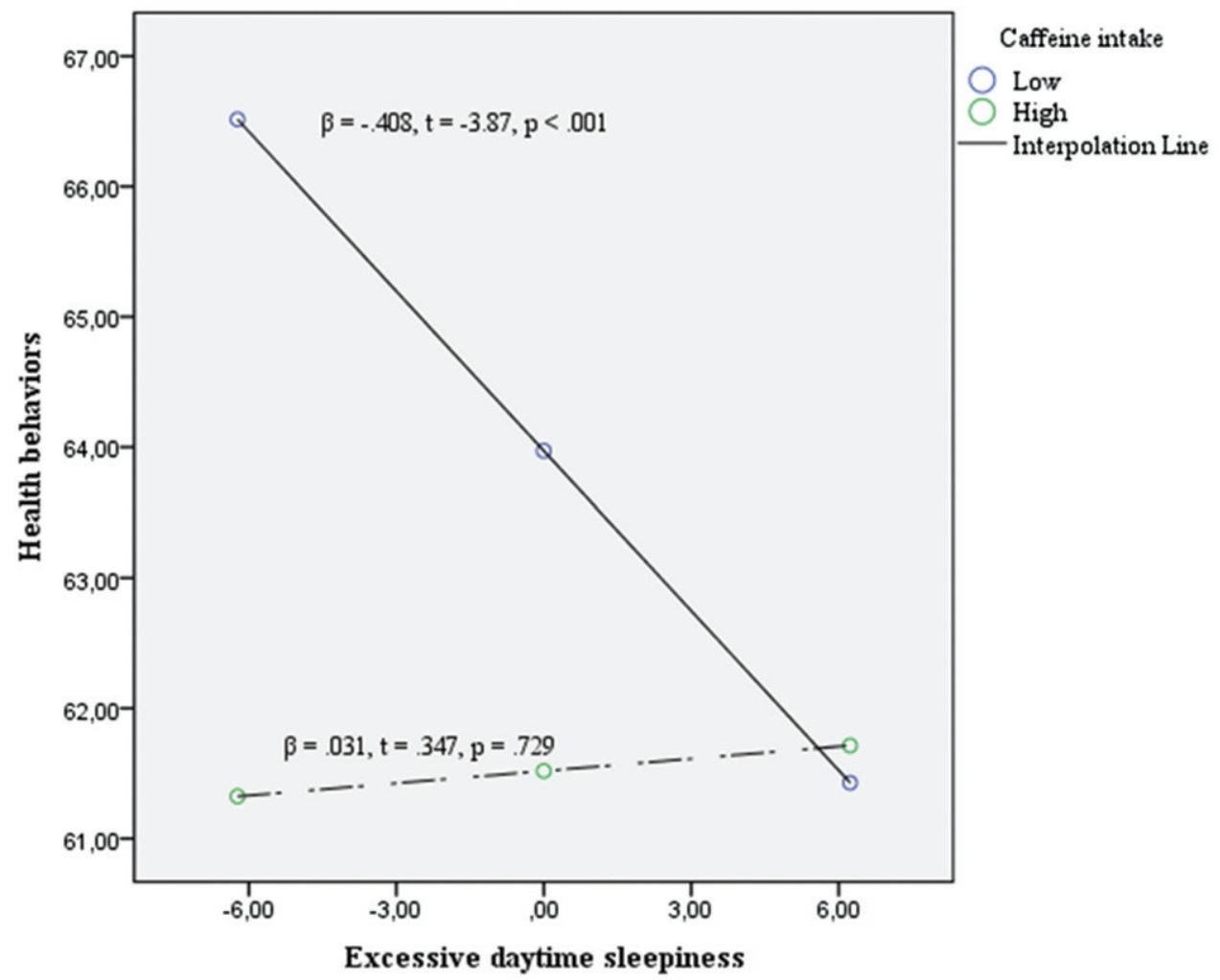

Figure 1. Caffeine intake as moderator between excursive sleepiness and health behavior. 
daytime sleepiness and worse family sleep behaviors were associated with fewer healthy behaviors.

Higher caffeine intake was significantly associated with lower sleep quality and fewer healthy behaviors. This result is in accordance with the literature that has shown students who consumed energy drinks reported poor sleep quality during the night followed by next-day tiredness (Patrick, Griffin, Huntley, \& Maggs, 2018). Also, high caffeine intake has been associated with worse health behaviors, such as high-fat diet (Temple et al., 2010). Moreover, higher caffeine intake was associated with worse family sleep behaviors. This result shows the importance that the family plays regarding the use of caffeine. Therefore, the family needs to be included in health behavior programs geared toward adolescents (Pearson et al., 2009).

Lower sleep quality and excessive daytime sleepiness were associated with worse family sleep behaviors. A study found that family dynamics (e.g., work-family conflict, family support) were associated with sleep quality in family members (Crain et al., 2014). The children reported higher sleep quality when the family had appropriate sleep routines and household rules (Buxton et al., 2015). Therefore, sleeprelated behaviors such as sleep hygiene, routines and healthy habits may impact not only the sleep quality at night, but also the consequences of poor sleep quality, which can be triggered by daytime sleepiness.

Lower sleep quality was associated with excessive daytime sleepiness. A literature review focused on the influence of sleep (quality, duration, sleepiness) in adolescents and children found poor sleep quality to be associated with increased daytime sleepiness (Dewald, Meijer, Oort, Kerkhof, \& Bögels, 2010). In the present study, lower sleep quality and excessive daytime sleepiness were associated with fewer healthy behaviors. A study that explored health behaviors around 3,400 students, from different ages and school levels, found associations among inadequate sleep, unhealthy behaviors (Chen et al., 2014), and risk behaviors such as smoking and driving after consuming alcohol (Owens \& Adolescent Sleep Working Group, 2014).

In the present study, the use of caffeine moderated the relationship between excessive daytime sleepiness and health behaviors being stronger when the use of caffeine was lower. A study showed a relationship between the consumption of caffeine and drowsiness, revealing that more than $75 \%$ of adolescents had a regular daily intake of caffeine (Kristjansson et al., 2011) and showing caffeine to be associated with other psychoactive drugs and daytime sleepiness. Also, a clinical review regarding the relationship between caffeine and sleepiness found that the regular use of caffeine was associated with daytime sleepiness and sleep disorders (Roehrs \& Roth, 2008). A recent pilot study also reported a relationship between the use of caffeine energy drinks and physiological effects, such as trouble sleeping, among adolescents (Jackson et al., 2013). A research aimed at identifying the impact of caffeine habits on sleep quality and daytime behavior found that excessive daytime sleepiness was a consequence of the caffeine consumption linked to the use of technology in adolescents (Calamaro et al., 2009).
Interestingly, when the caffeine intake was lower, the negative relationship was stronger, probably because adolescents who drank less caffeine may be more concerned with their health and, as a result, feel more the impact of excessive daytime sleepiness on their lifestyle. Future studies should pursue this hypothesis.

In this study, caffeine intake was not a moderator in the relationship between family sleep behaviors and sleep quality or health behaviors neither between daytime sleepiness and sleep quality. The literature has found poor sleep quality related to less health behaviors such as regular exercise and healthy diet (Chen, Wang, \& Jeng, 2006; Owens \& Adolescent Sleep Working Group, 2014). The fact that the relationship between family sleep behaviors and sleep quality as well as between family sleep behaviors and health behaviors was significant, but not moderated by caffeine intake, suggests the importance of family sleep behaviors on adolescents' health behaviors regardless of their use of caffeine (Wu, Tao, Zhang, \& Tao, 2008). The same holds true for the importance of daytime sleepiness on sleep quality regardless of the adolescent's use of caffeine.

\section{Limitations}

This study has several limitations, such as the cross-sectional design that does not allow making causal inferences and the exclusive use of self-report measures. When data collection took place, the adolescent version of PSQI was not published. Therefore, the present results need to be addressed cautiously. Future research should employ longitudinal designs to study the impact of caffeine intake on adolescents' sleep, as they grow older and analyze how caffeine intake will predict sleep disorders in adulthood.

\section{Conclusion and implications}

The present study found caffeine intake associated with worse family sleep behaviors and lower sleep quality and fewer healthy behaviors. Furthermore, the role of caffeine was a moderator in the relationship between excessive sleepiness and health behaviors. The risks of caffeine used by adolescents are still underestimated by health professionals and even by the general population (Jackson et al., 2013). This is often due to the fact that, culturally, caffeine is not seen as a psychoactive substance that causes addiction and negatively impacts adolescents. However, the use of caffeine intake is increasing among youngsters and, therefore, health education programs need to help adolescents decrease their consumption given its important role in the relationship between sleep and health behaviors. Teachers and family members should, therefore, be aware of adolescents' daytime sleepiness and refer teenagers to a pediatrician or a general health professional for a sleep assessment that should take into consideration the teenagers' caffeine intake. Also, in order to promote healthy behaviors, intervention programs should include ways to help adolescents decrease their caffeine intake, taking into consideration the 
adolescents' family behaviors. Therefore, intervention should be family centered.

\section{Funding}

The present research had a support of Erasmus Mundus (Lot 15) EMUNDUS15 Financial Agreement reference 20991670/001-001-ECW with a scholarship to the first author.

\section{Disclosure}

No authors present any conflict of interest.

\section{ORCID}

Sílvia Pucci (DD http://orcid.org/0000-0003-2258-007X

M. Graça Pereira (D) http://orcid.org/0000-0001-7987-2562

\section{References}

Bartel, K. A., Gradisar, M., \& Williamson, P. (2015). Protective and risk factors for adolescent sleep: A meta-analytic review. Sleep Med Rev, 21, 72-85. doi:10.1016/j.smrv.2014.08.002

Berge, J. M., Wall, M., Bauer, K. W., \& Neumark-Sztainer, D. (2010). Parenting characteristics in the home environment and adolescent overweight: A latent class analysis. Obesity, 18(4), 818-825. doi: 10.1038/oby.2009.324

Bernstein, G. A., Carroll, M. E., Thuras, P. D., Cosgrove, K. P., \& Roth, M. E. (2002). Caffeine dependence in teenagers. Drug Alcohol Depend, 66(1), 1-6. doi:10.1016/S0376-8716(01)00181-8

Billings, T., Bangash, O., \& Berg-Cross, L. (2010). Predicting sleep problems in adolescents: Validation of the family sleep questionnaire. Washington, DC: Howard University.

Billings, T., \& Berg-Cross, L. (2010). The modified Epworth sleepiness scale for adolescents: A brief measure of daytime sleepiness in adolescents. Washington, DC: Howard University.

Buxton, O. M., Chang, A. M., Spilsbury, J. C., Bos, T., Emsellem, H., \& Knutson, K. L. (2015). Sleep in the modern family: Protective family routines for child and adolescent sleep. Sleep Health: Journal of the National Sleep Foundation, 1(1), 15-27. doi:10.1016/ j.sleh.2014.12.002

Buysse, D. J., Reynolds, C. F., Monk, T. H., Berman, S. R., \& Kupfer, D. J. (1989). The Pittsburgh Sleep Quality Index: A new instrument for psychiatric practice and research. Psychiatry Research, 28(2), 193-213. doi:10.1016/0165-1781(89)90047-4

Calamaro, C. J., Mason, T. B., \& Ratcliffe, S. J. (2009). Adolescents living the 24/7 lifestyle: Effects of caffeine and technology on sleep duration and daytime functioning. Pediatrics, 123(6), 1005-1010. doi:10.1542/peds.2008-3641

Chen, G., Ratcliffe, J., Olds, T., Magarey, A., Jones, M., \& Leslie, E. (2014). BMI, health behaviors, and quality of life in children and adolescents: A school-based study. Pediatrics, 133(4), e868-e874. doi:10.1542/peds.2013-0622

Chen, M. Y., Wang, E. K., \& Jeng, Y. J. (2006). Adequate sleep among adolescents is positively associated with health status and healthrelated behaviors. BMC Public Health, 6(1), 59. doi:10.1186/14712458-6-59

Crain, T. L., Hammer, L. B., Bodner, T., Kossek, E. E., Moen, P., Lilienthal, R., \& Buxton, O. M. (2014). Work-family conflict, family-supportive supervisor behaviors (FSSB), and sleep outcomes. Journal of Occupational Health Psychology, 19(2), 155. doi:10.1037/ a0036010

DeVellis, R. F. (1991). Scale development. Theory and applications. London: Sage Publications.
Dewald, J. F., Meijer, A. M., Oort, F. J., Kerkhof, G. A., \& Bögels, S. M. (2010). The influence of sleep quality, sleep duration and sleepiness on school performance in children and adolescents: A meta-analytic review. Sleep Medicine Reviews, 14(3), 179-189. doi: 10.1016/j.smrv.2009.10.004

Hayes, A. F. (2017). An Introduction to mediation, moderation, and conditional process analysis: A regression-based approach. New York, NY: Guilford Publications. doi:10.1111/jedm.12050

Jackson, D. A. E., Cotter, B. V., Merchant, R. C., Babu, K. M., Baird, J. R., Nirenberg, T., \& Linakis, J. G. (2013). Behavioral and physiologic adverse effects in adolescent and young adult emergency department patients reporting use of energy drinks and caffeine. Clinical Toxicology, 51(7), 557-565. doi:10.3109/15563650.2013.820311

Juliano, L. M., \& Griffiths, R. R. (2004). A critical review of caffeine withdrawal: Empirical validation of symptoms and signs, incidence, severity, and associated features. Psychopharmacology, 176(1), 1-29. doi:10.1007/s00213-004-2000-x

Kristjansson, A. L., Sigfusdottir, I. D., Allegrante, J. P., \& James, J. E. (2011). Adolescent caffeine consumption, daytime sleepiness, and anger. J Caffeine Res, 1(1), 75-82. doi:10.1089/jcr.2011.0008

Maes, S., Leventhal, H., \& de Ridder, D. T. (1996). Coping with chronic diseases. London: Wiley.

Neumark-Sztainer, D., Story, M., Hannan, P. J., Perry, C. L., \& Irving, L. M. (2002). Weight-related concerns and behaviors among overweight and nonoverweight adolescents: Implications for preventing weight-related disorders. Archives of Pediatrics \& Adolescent Medicine, 156(2), 171-178. doi:10.1001/archpedi.156.2.171

Owens, J., \& Adolescent Sleep Working Group. (2014). Insufficient sleep in adolescents and young adults: An update on causes and consequences. Pediatrics, 134(3), e921-e932. doi:10.1542/peds.20141696

Owens, J., Wang, G., Lewin, D., Skora, E., \& Baylor, A. (2017). Association between short sleep duration and risk behavior factors in middle school students. Sleep, 40(1), e04-e04. doi:10.1093/sleep/ zsw004

Patrick, M. E., Griffin, J., Huntley, E. D., \& Maggs, J. L. (2018). Energy drinks and binge drinking predict college students' sleep quantity, quality, and tiredness. Behavioral Sleep Medicine, 16(1), 92-105. doi: 10.1080/15402002.2016.1173554

Pearson, N., Biddle, S. J., \& Gorely, T. (2009). Family correlates of breakfast consumption among children and adolescents. A systematic review. Appetite, 52(1), 1-7. doi:10.1016/j.appet.2008.08.006

Pedras, C. S. (2008). Variáveis de Saúde, Familiares e de Psicopatologia em Filhos de Veteranos da Guerra Colonial Portuguesa. [Health, Family and Psychopatholofy Variables in the Offspring of Portuguese Colonial War Veterans] . Master Thesis. University of Minho, Scool of Psychology. Braga, Portugal.

Reissig, C. J., Strain, E. C., \& Griffiths, R. R. (2009). Caffeinated energy drinks-a growing problem. Drug Alcohol Depend, 99(1-3), 1-10. doi:10.1016/j.drugalcdep.2008.08.001

Roehrs, T., \& Roth, T. (2008). Caffeine: Sleep and daytime sleepiness. Sleep. Med Rev, 12(2), 153-162. doi:10.1016/j.smrv.2007.07.004

Temple, J. L. (2009). Caffeine use in children: What we know, what we have left to learn, and why we should worry. Neuroscience Biobehavior Review, 33(6), 793-806. doi:10.1016/j.neubiorev.2009.01.001

Temple, J. L., Dewey, A. M., \& Briatico, L. N. (2010). Effects of acute caffeine administration on adolescents. Experimental and Clinical Psychopharmacology, 18(6), 510. doi:10.1037/a0021651

Von Elm, E., Altman, D. G., Egger, M., Pocock, S. J., Gøtzsche, P. C., \& Vandenbroucke, J. P. (2007). The Strengthening the Reporting of Observational Studies in Epidemiology (STROBE) statement: Guidelines for reporting observational studies. Preventive Medicine, 45(4), 247-251. doi:10.1016/S0140-6736(07)61602-x

Wu, X., Tao, S., Zhang, S., \& Tao, F. (2008). Low physical activity, high screen time can increase the risks of mental health problems and poor sleep quality among Chinese college students. Plos One, 24(9), e0119607. doi:10.1371/journal.pone.0119607 
Copyright of Journal of Child \& Adolescent Substance Abuse is the property of Taylor \& Francis Ltd and its content may not be copied or emailed to multiple sites or posted to a listserv without the copyright holder's express written permission. However, users may print, download, or email articles for individual use. 